On constate une perte importante de $\mathrm{P}^{2} \mathrm{O}^{5}$ variant dans nos essais de 12 à $22 \%$ de l'acide phosphorique du phosphate tricalcique mis en œuvre. La perte croît avec la quantité d'acide sulfurique utilisé.

Notre hypotbèse se trouve donc vérifiée.

C'est la condamnation de la technique des cendres sulfuriques, au moins dans les produits contenant de l'acide phosphorique soit organique, soit même combiné à une base terreuse.

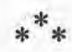

\title{
Conclusion
}

La meilleure méthode pour la détermination exacte des matières minérales de la caséine lactique nous paraît être celle que nous avons antérieurement décrite (Le Lait, novembre 1933, p. 1081), mais à la condition de remplacer la solution d'acétate de calcium par une solution d'acétate de magnésium.

\section{LES PHOSPHATIDES DANS LE RÉSIDU DU BEURRE}

\section{par le Dr BRuno REWALD}

de Londres

La proportion de phosphatides dans le beurre n'est pas très élevée; mais la présence de ces phosphatides est importante, parce que nous savons qu'elles ont une influence nette sur les propriétés de cette matière grasse si appréciée. Dans quelques pays du Continent, surtout en Suisse, on utilise un procédé spécial pour séparer la matière grasse pure du beurre de tous les autres composants normaux du beurre : eau, caséine, sucre de lait, sel, etc. Ce procédé consiste à chauffer la matière grasse du beurre à une température telle que toute l'eau s'évapore. Cette opération effectuée, les autres sous-produits deviennent absolument insolubles; toute la structure du "beurre" est modifiée, l'émulsion est détruite et les substances non huileuses se séparent au fond. La matière grasse du beurre claire et exempte d'eau se conserve très longtemps sans se détériorer si on la conserve en boîtes de fer-blanc ou autres récipients.

Le résidu consiste en un produit brun sombre qui ressemble à de la graisse et qui contient naturellement toutes les "impuretés " du beurre ci-dessus mentionnées, mais contient aussi encore une certaine proportion de matière grasse du beurre et, en outre, la plupart des phosphatides qui sont séparées et précipitées. La température élevée à laquelle ce résidu est obtenu a naturellement aussi une influence sur la composition de ses différents composants, mais 
il semble résulter de nos recherches que la plupart des phosphatides n'ont pas encore été modifiées.

Ce résidu est naturellement un milieu bien meilleur pour l'extraction des phosphatides que le beurre ou le lait condensé ou le lait sec. La séparation des matières grasses et des phosphatides peut être effectuée de la manière habituelle, par extraction à l'aide d'un dissolvant; mais ces extractions doivent être répétées souvent avant que tous les produits solubles dans le dissolvant soient épuisés. Au moins 20 extractions sont nécessaires et il est possible que même de petites proportions restent encore dans le résidu (1).

On traite d'abord avec de l'éther de pétrole (point d'ébullition entre $54^{\circ}$ et $60^{\circ}$ C.) 500 grammes du résidu de beurre. La quantité totale de l'extrait obtenu fut de $80 \mathrm{gr}$. 4, soit $16,1 \%$. Par addition d'acétone, la matière grasse du beurre soluble qui ne contient que des traces de phosphatides, peut être séparée. La quantité totale de phosphatides fut de 26 gr. 3, soit $5,26 \%$ du residu. De cette façon, il est facile d'obtenir une certaine quantité de ces phosphatides. Dans ce cas aussi, les phosphatides sont un mélange de lécithine pure, de céphaline pure et d'un produit qu'on peut classer entre ces deux produits bien connus. Par un traitement à l'alcool pur $(99 \%)$, la lécithine se dissout et peut être séparée du résidu. En faisant bouillir ce résidu avec de l'alcool, et en filtrant, une certaine proportion est soluble qui se sépare en une masse solide par refroidissement et une quantité plus grande reste insoluble dans l'alcool chaud et l'alcool froid (céphaline).

De cette façon, 12 gr. $9=2,58 \%$ de céphaline sont séparés et 9 gr. $41=1,88 \%$ de lécithine, ainsi que $4 \mathrm{gr} .02=0,88 \%$ du produit soluble à chaud et insoluble à froid. On sait que la plupart des phosphatides dans les produits animaux, par exemple dans le jaune d'ceuf, ne peuvent pas être extraites de la manière habituelle avec seulement de l'éther de pétrole. Les phosphatides forment une sorte de combinaison "physique" avee les protéines, et ce n'est qu'en utilisant de l'alcool que ces phosphatides peuvent être séparées des protéines. C'est pourquoi un second dissolvant doit être utilisé pour extraire toutes les phosphatides présentes. Mais très souvent existent des phosphatides insolubles dans l'alcool, et c'est pourquoi il est recommandé d'employer un mélange de dissolvants, l'alcool pour la séparation et le benzol, ou un dissolvant analogue, pour dissoudre ees phosphatides non "libres".

Un mélange d'alecol et de benzol ( 80 pour 20) fut employé pour une nouvelle extraction de ce résidu de beurre. En présence de l'alcool, d'autres composants, spécialement les sucres, sont aussi

(1) Ce résidu nous a été aimablement envoyé par le Dr Ritrer, de Liebefeld (Berne). 
solubles; e'est pourquoi il est nécessaire de purifier cet extrait, ce qui peut être fait en utilisant de l'éther de pétrole comme dissolvant du résidu desséché dans le vide. De cette façon, on a pu séparer encore $25 \mathrm{gr} .81=5,16 \%$ de produits analogues à la graisse. En utilisant le même procédé que celui mentionné ci-dessus, les chiffres ci-dessous indiquent la composition de cet extrait par l'alcool et le benzol :

$\begin{array}{lcc} & \text { Grammes } & \% \\ \text { Soluble dans l'acétone (matière grasse) } \ldots \ldots & 7,4 & 1,48 \\ \text { Insoluble dans l'alcool (éphaline) } \ldots \ldots \ldots \ldots & 3,63 & 0,72 \\ \begin{array}{l}\text { Soluble dans l'aleool (lécithine) } \ldots \ldots \ldots \ldots \\ \text { Soluble dans l'alcool à chaud, insoluble à }\end{array} & 13,045 & 2,64 \\ \quad \text { froid } \ldots \ldots \ldots \ldots \ldots \ldots \ldots \ldots \ldots \ldots \ldots \ldots & 1,735 & 0,35\end{array}$

On voit par ces chiffres qu'un fort pourcentage de ces phosphatides est dans l'état appelé " en liaison "; mais que le chauffage de la matière grasse du beurre et aussi du résidu de beurre semblent avoir influencé à un certain point la composition des phosphatides, car autrement le pourcentage des phosphatides "liées" en comparaison des phosphatides "libres" aurait dû́ être bien plus élevé.

Il est très intéressant de comparer la solubilité des phosphatides dans les deux dissolvants. En tout, il y a :

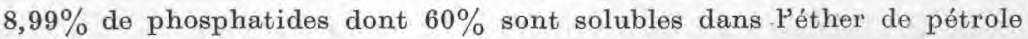
et $40 \%$ solubles dans l'alcool et le benzol.

$3,30 \%$ de céphaline dont $78 \%$ sont solubles dans l'éther de pétrole et $22 \%$ solubles dans l'alcool et le benzol.

$4,52 \%$ de lécithine dont $42 \%$ sont solubles dans l'éther de pétrole et $58 \%$ solubles dans l'alcool et le benzol.

$1,15 \%$ soluble à chaud, insoluble à froid dont $60 \%$ sont solubles dans l'éther de pétrole et $40 \%$ solubles dans l'alcool et le benzol.

Il est remarquable que l'extraction par l'éther de pétrole donne un rendement beaucoup moindre avec la lécithine qu'avec la céphaline.

Il y a donc, d'après ces résultats, dans la quantité totale de phosphatides existant dans le résidu de beurre, et par conséquent existant aussi dans le beurre lui-même et dans le lait :

$36 \%$ de céphaline (insoluble dans l'alcool).

$50 \%$ de lécithine (soluble dans l'alcool),

$14 \%$ soluble à chaud et insoluble à froid.

Les six échantillons différents de phosphatides ont été analysés et on a calculé la teneur en $\mathrm{P}$ et en $\mathrm{N}$ ainsi que le rapport $\mathrm{P} / \mathrm{N}$. Les chiffres sont les suivants : 
Extraction à l'éther de pétrole.

\begin{tabular}{|c|c|c|c|}
\hline Insoluble dans l'aleool (céphaline) & 3,67 & 1,52 & $1 / 1,0$ \\
\hline Soluble dans l'alcool (lécithine) & 2,67 & 1,11 & $1 / 0,9$ \\
\hline Soluble à chaud, insoluble à froid . & 3,20 & 1,35 & $1 / 1,1$ \\
\hline \multicolumn{4}{|l|}{ xtraction à l'alcool-benzol. } \\
\hline Insoluble dans l'aleool (céphaline) & 2,69 & 1,21 & $1 / 1,0$ \\
\hline Soluble dans l'alcool (lécithine) & 2,38 & 1,20 & $1 / 1,1$ \\
\hline Soluble à chaud, insoluble à froid . & - & - & . \\
\hline
\end{tabular}

La plupart des phosphatides n'étaient pas pures ; une céphaline pure a environ $4,1 \%$ de $\mathrm{P}$ et une lécithine pure $3,8 \%$ de $\mathrm{P}$. Il semble donc que le chauffage ait eu quelque influence; toutes les phosphatides étaient de couleur très sombre et il est possible que quelques petites quantités de sucre ou de sucre brûlé soient présentes. Il ést très ditficile d'éliminer les dernières traces des sous-produits. D'un autre côté, il est remarquable que le rapport $\mathrm{P} / \mathrm{N}$ ait été dans tous les cas exactement ou presque exactement $1 / 1$, ce qui signifie que toutes les phosphatides séparées sont des monoaminomonophosphatides.

Ces chiffres correspondent à ceux d'une étude précédente (1) dans laquelle est donnée également une revue des études précédentes sur les phosphatides du lait. On avait extrait du lait sec les phosphatides avec seulement de l'alcool et de l'alcool et du benzol. La proportion de lécithine était de $58,3 \%$, celle de céphaline : $41,7 \%$. On n'avait pas procédé à d'autres séparations.

Il ressort de ces recherches que les phosphatides existent aussi dans le lait et dans le beurre sous deux formes différentes, sous forme de phosphatides "libres", et de phosphatides "liées»; que dans chaque cas il existe au moins trois types différents de phosphatides; et que toutes les phosphatides extraites sont du type monoaminomonophosphatide. Il doit être mentionné que la matière première, le résidu de beurre, peut avoir subi une certaine modification qui peut avoir influencé jusqu'à un certain point la composition des phosphatides.

\section{LE DOSAGE DE LA MATIÈRE GRASSE DANS LES FROMAGES (2)}

\section{par DANIEL FLORENTIN}

Ce sujet paraît, a priori, complètement épuisé, tout ayant été dit sur la question.

Tout récemment, M. PIen, dans la Revue Le Lait (t. XVII,

(1) B. REWALD. Le Lait, t. XVII, mars 1937, p. 225-229.

(2) Annales des Falsifications et des. Fraudeৎ, no 355-356, juillet-août 1938, p. 351. 